\title{
Exploring food insecurity and obesity in Dutch families: a mediation analysis
}

\section{Abstract}

Food insecurity is related to risk of adverse health outcomes such as obesity, but the factors underlying this association are still unclear. We aimed to assess the association between food insecurity status and obesity, and to explore potential mediation by sociodemographic and lifestyle factors.

This cross-sectional study was conducted among 250 participants in a deprived urban area in The Hague, the Netherlands. Data on sociodemographic and lifestyle factors, food insecurity status and diet quality were collected using questionnaires. Diet quality was determined based on current national dietary guidelines. Regression analyses were performed to explore the association between food insecurity and BMI status. To assess the role of potential mediators, mediation analyses were performed to estimate the total effect, direct effect, and indirect effect and proportion of the total effect mediated of the food insecurity status-obesity association through potential mediators, crude and controlling for confounders.

The overall prevalence of food insecurity was 26 percent. Food insecurity was associated with obesity $(\mathrm{OR}=2.49,95 \% \mathrm{CI} 1.16$, 5.33 ), but not with overweight (OR $1.15,95 \%$ CI $0.54,2.45)$. The association between food insecurity status and obesity was partially mediated by living situation (proportion mediated: $15.3 \%$ ), and general health status (19.1\%) (consistent mediation). Diet quality was an inconsistent partial mediator (-17.7\%). After controlling for confounders, smoking status became an inconsistent partial mediator $(-15.8 \%)$, and living situation and diet quality remained partial mediators.

Food insecurity is associated with obesity and this association is partially mediated by living situation, and inconsistently partially mediated by diet quality and smoking status when controlling for confounders. Future studies are warranted to test other potential mediators that might underlie the association between food insecurity and obesity. Our findings emphasize the importance of preventing food insecurity to achieve public health goals. In addition, food insecurity and its potential consequences (i.e. an unhealthy lifestyle such as smoking, poor health, and unfavorable living situations) need to be taken into account in obesity prevention programs and policies.

\section{Conflict of Interest}

There is no conflict of interest 